\title{
Epicuticular Wax and White Pine Blister Rust Resistance in Resistant and Susceptible Selections of Eastern White Pine (Pinus strobus)
}

\author{
Jason A. Smith, Robert A. Blanchette, Todd A. Burnes, Jeffrey H. Gillman, and Andrew J. David
}

First, second, and third authors: Department of Plant Pathology, University of Minnesota, 495 Borlaug Hall, 1991 Upper Buford Cir., St. Paul, MN 55108; fourth author: Department of Horticultural Science, University of Minnesota, 305 Alderman Hall, 1970 Folwell Avenue, St. Paul, MN 55108; and fifth author: Department of Forest Resources, University of Minnesota, North Central Research and Outreach Center, 1861 East Highway 169, Grand Rapids, MN 55744.

Accepted for publication 15 October 2005.

\begin{abstract}
Smith, J. A., Blanchette, R. A., Burnes, T. A., Gillman, J. H., and David, A. J. 2006. Epicuticular wax and white pine blister rust resistance in resistant and susceptible selections of eastern white pine (Pinus strobus). Phytopathology 96:171-177.

Epicuticular wax on needles was evaluated for its influence on Cronartium ribicola infection of resistant and susceptible selections of Pinus strobus. Environmental scanning electron microscopy comparisons revealed that needles from a resistant selection of eastern white pine, P327, had a significantly higher percentage of stomata that were occluded with wax, fewer basidiospores germinating at $48 \mathrm{~h}$ after inoculation, and fewer germ tubes penetrating stomata than needles from a susceptible selection

cluded with wax compared with needles from seedlings that developed symptoms. In experiments where epicuticular waxes were removed from needles before seedlings were infected, resistant seedlings without wax developed approximately the same number of infection spots (as measured by spot index) as susceptible seedlings with wax intact. Gas chromatography/mass spectrometry comparisons of extracted epicuticular waxes revealed several peaks that were specific to P327 and not found in susceptible H111 suggesting biochemical differences in wax composition. These results implicate the role of epicuticular waxes as a resistance mechanism in $P$. strobus selection P327 and suggest a role for waxes in reducing spore germination and subsequent infection through stomatal openings.
\end{abstract} H111. In addition, needles from seedlings that failed to develop symptoms 6 weeks after inoculation, from a cross between P327 and susceptible parent H109, had a significantly higher percentage of stomata oc-
Additional keywords: disease resistance, foliar cuticle, hyphae, pine needles.
White pine blister rust (WPBR), caused by Cronartium ribicola, is a serious disease of five-needled pines (white pines) in North America $(9,11)$. The pathogen was introduced to eastern and western North America on infected Pinus strobus (eastern white pine) seedlings shipped from Europe around 1900 and has caused widespread damage to many white pine populations throughout North America $(7,9)$.

Although the majority of white pines in North America are very susceptible to the disease, resistant individuals have been found in several species including $P$. strobus $(5,8,14,16,18)$. However, little is known about what factors contribute to resistance for WPBR, especially in $P$. strobus. In a recent study, Jurgens et al. (7) used histology to examine infections in resistant and susceptible seedlings of $P$. strobus and determined that fungal growth was arrested in resistant individuals. This was accompanied by accumulation of phenolic compounds around the infection site and death of surrounding cells in a hypersensitive-like response. On resistant seedlings, infections and needle lesions were often much slower to develop than on susceptible seedlings and often these infections stopped developing completely. In addition, it was also observed that fewer spots developed on open-pollinated seedlings of resistant selection P327, a finding that had been observed by Patton (10) and Patton and Spear (15) more than 20 years earlier and had been termed "reduced needle-lesion frequency."

The initial infection process for WPBR has been well documented $(1,3,4,6,13)$. Germinating basidiospores produce germ

Corresponding author: J. A. Smith: E-mail address: smit1278@umn.edu

DOI: 10.1094/PHYTO-96-0171

(c) 2006 The American Phytopathological Society tubes that enter the outer stomatal chamber and penetrate through apertures between guard cells. Upon entry, a substomatal vesicle is produced and an infection hypha develops into mycelium that penetrates the mesophyll cells $(6,13)$.

Patton and Spear (15) looked at stomatal influences on infection and suggested that wax occlusion of stomata may be a reason for failed infections after artificial inoculations. Patton (11) also speculated that epicuticular wax may be responsible for the reduction in infections on older needles versus younger needles (or primary versus secondary needles). However, no systematic comparison of resistant and susceptible seedlings was performed largely because at the time preparation techniques for scanning electron microscopy altered the appearance of epicuticular wax, making accurate comparisons difficult (15).

In the process of screening $P$. strobus families for resistance to WPBR at the University of Minnesota, it was observed that progeny of P327 developed fewer and smaller spots compared with susceptible controls. It was also observed that parent clone P327 appeared more silvery in color compared with wild type $P$. strobus - a result of different epicuticular wax characteristics. These two observations suggest that epicuticular waxes in P327 may have a role in preventing infection by $C$. ribicola; therefore, this study focused on comparing this seed-source with susceptible controls. Although Heimburger (5) selected trees with superior silvicultural traits, his selections (H109 and H111) used in this study serve as susceptible controls since they were determined to be highly susceptible to infection in previous studies by Jurgens et al. (7).

The goal of this study was to determine the role epicuticular wax might play in reducing infection frequency. Environmental electron microscopy was used to investigate differences in wax 
occlusion of stomata, spore germination, and subsequent entry into stomata. In addition, gas chromatography/mass spectrometry (GC/MS) analyses were used to determine if waxes from resistant and susceptible needles differ in biochemical composition.

\section{MATERIALS AND METHODS}

Plant materials and macroscopic evaluations. Grafts and seedlings from trees selected by Patton (14) and Heimburger (5) and growing in the USDA Forest Service, Oconto River Seed Orchard (ORSO) in White Lake, WI, were used for the studies reported here. P. strobus selection P327 was selected because it was phenotypically resistant to WPBR in the field and grafts of this selection were planted (in the 1960s) and are available at ORSO.

One-year-old scions from the grafted clones at ORSO were grafted onto potted wild-type 2-0 seedlings of Lake States origin from a commercial source. Grafts were grown for 1 year before being used in experiments. Seed was collected from cones that resulted from controlled crosses of grafts of $P$. strobus selections at ORSO. The seed was cold-moist stratified for at least 40 days and planted in artificial potting mix in 1-in.-diameter tubes. Seedlings were grown in the greenhouse under artificial lights for 8 weeks before being inoculated, and were fertilized with 15-15-15 watersoluble fertilizer every 4 weeks.

Quantitative comparisons of epicuticular wax. Quantitative comparisons of epicuticular wax from primary and secondary needles of resistant and susceptible $P$. strobus selections were completed. Eight-week-old primary needles from 12 seedlings each of P327 $\times$ P327 and H111 $\times$ H111 and secondary needles from clones of P327 and H111 were used for comparisons. Twelve needles per seedling were collected from greenhouse-grown plants and brought to the laboratory. Epicuticular wax was removed by placing the needles in preweighed 5-ml glass tubes filled with chloroform. After $2 \mathrm{~min}$, the needles were removed and the tubes were placed in a fume hood for evaporation. After $24 \mathrm{~h}$, the tubes were reweighed and dry weight was calculated. Means were calculated for the samples (mean for each seedling) and statistical analyses were performed using one-way analysis of variance (ANOVA) and Waller-Duncan multiple means comparison using Statistical Analysis Software (SPSS, Inc., Chicago, IL). Subgroups were considered significantly different at the $\alpha=0.05$ level.

Stomatal wax occlusion comparisons. To determine if $P$. strobus families differ in the percentage of stomata that are occluded with wax, needles were compared using environmental scanning electron microscopy (ESEM). Eight needles were collected from each of eight plants in the following categories: 2-year-old H111 grafts (secondary needles only), 8-week-old H111 × H111 seedlings (primary needles only), 2-year-old P327 grafts (secondary needles only), and 8-week-old P327 × P327 seedlings (primary needles only).

Three segments ( $1 \mathrm{~cm}$ from the middle and two ends) of each needle were prepared for ESEM and attached to stubs with a thin layer of finger nail polish. Stubs were coated with gold and placed in the low-vacuum, variable-pressure chamber of the Hitachi S3500 Scanning Electron Microscope and photographed with a digital camera at approximately $\times 800$ to $\times 1,800$ magnification. Images were collected at three predetermined locations (using the $x$ axis), and data were pooled from these sampling points. In each field of view, the total number of stomata and the number of stomata occluded with wax were counted. Stomata were considered occluded if there were no gaps in the wax plug greater than $5 \mu \mathrm{m}$. The means for the eight needles collected from each plant were pooled. Statistical analyses were performed using one-way ANOVA and Waller-Duncan multiple means comparison using Statistical Analysis Software (SPSS, Inc.). Means (needle type/seed-source) were considered significantly different at the $\alpha=0.05$ level.
ESEM was used to investigate differences in stomatal wax occlusion between infected and uninfected seedlings resulting from a cross between P327 (resistant) and H109 (susceptible). The basidiospore inoculation protocol of Jurgens et al. (7) was used to artificially inoculate these seedlings. Primary needles with successful infections (showing typical needle spots) and needles not infected were removed at 4-weeks postinoculation from inoculated 6-month-old seedlings of P327 × H109. Primary needles from mock-inoculated seedlings of open-pollinated P327 and H109 were also used for ESEM evaluation of stomatal wax occlusion using the same methods as described previously. For each seedling group, 10 primary needles were selected from each of 33 seedlings, providing 330 replicates for each of the seedling groups. Means were calculated for each seedling group and statistical analyses were performed using one-way ANOVA and WallerDuncan multiple means comparison using Statistical Analysis Software (SPSS, Inc.). Means were considered significantly different at the $\alpha=0.05$ level.

Spore germination and stomatal penetration. Spore germination and stomatal penetration differences between $P$. strobus families P327 and H111 were determined using ESEM. Needles from $P$. wallichiana (co-evolved resistant host) and Metasequoia glyptostroboides (nonhost) were used for comparison. Three needles from each of 10 plants per group were collected and transferred to ESEM stubs with a thin layer of finger nail polish to hold samples in place. The following were used for analysis: secondary needles from 2-year-old P327 graft, primary needles from seedlings of P327 $\times$ P327, secondary needles from H111 graft, primary needles from seedlings of $\mathrm{H} 111 \times \mathrm{H} 111$, secondary needles from seedlings of $P$. wallichiana, and secondary needles from seedlings of $M$. glyptostroboides (nonhost). Care was taken to only touch the basal tip of the needle and ensure the two sides with stomata were placed facing up. The stubs were then placed in plastic EM stub boxes with a telia-bearing $R$. nigrum leaf adhered to the inside cover of the box with petroleum jelly. The $R$. nigrum samples were inoculated with a strain of $C$. ribicola from Wisconsin using the protocol described previously in Jurgens et al. (7). Wet filter paper was placed under the stubs, and after misting the Ribes leaves and P. strobus needles with distilled water, the box was sealed shut with Parafilm and placed in a dark growth chamber at $18^{\circ} \mathrm{C}$ for $48 \mathrm{~h}$. At approximately 8-h intervals, the stubs were rotated within the box to ensure equal distribution of basidiospores. Using the same ESEM methods described previously, the samples were evaluated and in each predetermined field of view (three per sample), the number of spores, the number of germinating spores, and the number of spores with hyphae penetrating stomata were counted. Data for each needle sample were pooled and means were calculated. Statistical analyses were performed using one-way ANOVA and Waller-Duncan multiple means comparison using Statistical Analysis Software (SPSS, Inc.). Means (P327 primary, P327 secondary, H111 primary, H111 secondary, etc.) were considered significantly different at the $\alpha=$ 0.05 level.

Wax removal inoculation. To examine whether wax removal influences infection of needles, $C$. ribicola inoculation experiments were done after seedlings were treated to remove epicuticular wax. Several methods were attempted to remove wax while avoiding damage to the mesophyll cells or causing the needles to scorch or become necrotic. After several attempts, a method was devised by using sterile Q-tips to swab needle surfaces with $50 \%$, $\mathrm{vol} / \mathrm{vol}$, solution of chloroform (in $95 \% \mathrm{ETOH}$ ). Immediately after brushing the needle surfaces with the Q-tip, the seedlings were rinsed in water to remove any excess chloroform. Twelve primary needles from each of eight 6-month-old P327 × P327 and H111 $\times$ H111 were treated to remove wax. Eight untreated seedlings of each were used as controls; eight of each seed-source were also mock-inoculated with healthy Ribes leaves. After seedlings were treated they were placed in a growth chamber with 80 to $90 \%$ hu- 
midity and held at $15^{\circ} \mathrm{C}$ until they were inoculated. Inoculation with $C$. ribicola followed the protocol described by Jurgens et al. (7). Needles from each seedling group were collected prior to inoculation and evaluated for wax occlusion of stomata using ESEM (same methods as described previously). After 6 weeks, seedlings were evaluated for symptoms. Three symptom evaluations were performed: spot number index, spot size index, and number of needles with spots. Indexing the number of spots per plant was done according to the following scale: index $0=0$ spots, index $1=1$ to 3 spots, index $2=4$ to 10 spots, index $3=11$ to 32 spots, and index $4=33$ to 100 spots per plant. For spot size index, the largest spot per seedling was ranked using the following scale: $0=$ no infection, $1=$ spot length of $>0$ to $2.0 \mathrm{~mm}, 2=$ spot length of $>2.0$ to $4.0 \mathrm{~mm}, 3=$ spot length of $>4.0$ to $8.0 \mathrm{~mm}$, and $4=$ spot length of $>8.0 \mathrm{~mm}$. The number of needles with spots was also counted for each seedling. Data were pooled for each treatment and measurement (spot index, number of needles infected, and spot size) and means were calculated. For each dependent variable, statistical analyses were performed using oneway ANOVA and Waller-Duncan multiple means comparison using Statistical Analysis Software (SPSS, Inc.). Means were considered significantly different at the $\alpha=0.05$ level.

GC/MS comparison of extracted needle wax. For a comparative study of the chemical components in wax from needles, wax was removed using chloroform as a solvent from $1 \mathrm{~g}$ each of primary needles from 6-month-old P327 $\times$ P327 and H111 $\times$ H111 and secondary needles of 2-year-old grafts of P327 and H111. The chloroform was placed in GC autosampler vials until they were analyzed. A Varian Star $3400 \mathrm{Cx}$ Ion Trap GC/MS (EI) (Varian Inc., Palo Alto, CA) fitted with a DB-wax column $(30 \mathrm{~m} \times$ $0.25 \mathrm{~mm}$ ) (Agilent Technologies, Palo Alto, CA) was used to analyze samples. The column temperature was $60^{\circ} \mathrm{C}$, the injector temperature was $220^{\circ} \mathrm{C}$, and the detector temperature was $230^{\circ} \mathrm{C}$. The oven was held at $60^{\circ} \mathrm{C}$ for $3 \mathrm{~min}$ and then increased to $225^{\circ} \mathrm{C}$ at a rate of $8^{\circ} \mathrm{C}$ per min for $20.62 \mathrm{~min}$ and held at $225^{\circ} \mathrm{C}$ for $12 \mathrm{~min}$. The chromatographs were compared and individual peaks were identified by comparing mass spectra to the library references.

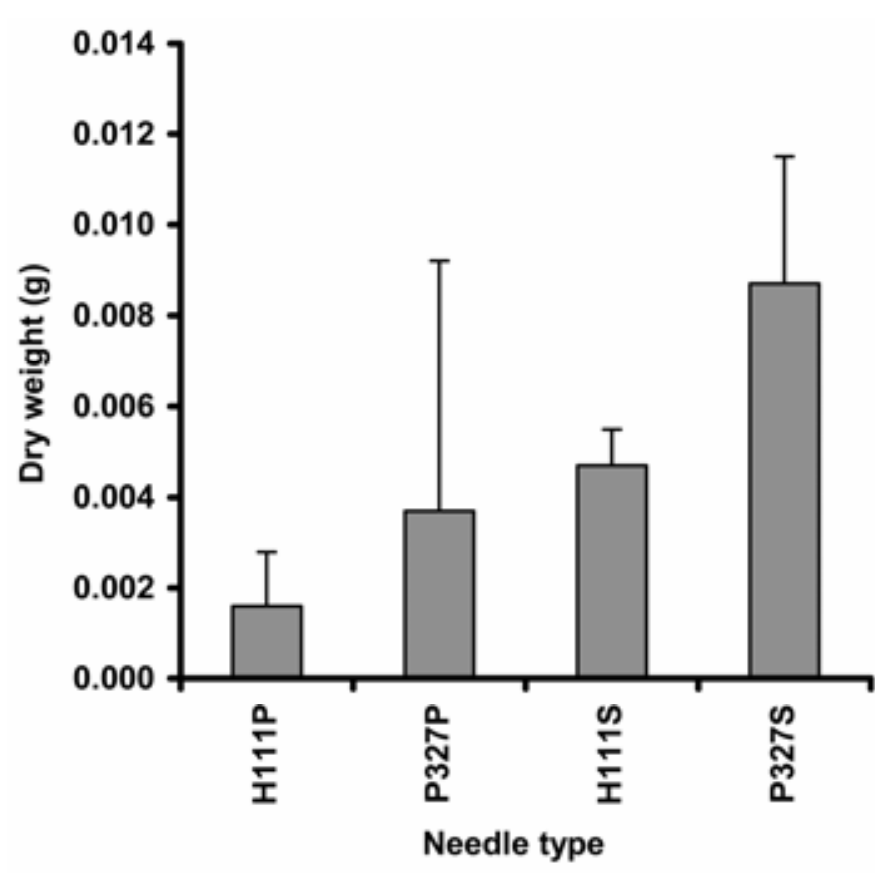

Fig. 1. Mean dry weight measurements $(\mathrm{g})$ of needle wax removed from $1 \mathrm{~g}$ of primary $(\mathrm{P})$ and secondary $(\mathrm{S})$ needles from seedlings of selfed crosses of white pine blister rust-susceptible and -resistant Pinus strobus selections H111 and P327, respectively. Error bars represent standard deviation for each group.

\section{RESULTS}

Quantitative comparisons of epicuticular wax. Although differences in dry weights of wax samples were not statistically significant $(\alpha=0.05)$, there was a trend of resistant family P327 having a greater amount of wax per gram of needle tissue (Fig. 1). Among primary needles, seedlings from the cross $\mathrm{H} 111 \times \mathrm{H} 111$ had a mean of $1.6 \times 10^{-3} \mathrm{~g}$ (standard deviation $[\mathrm{SD}]=1.9 \times$ $10^{-3} \mathrm{~g}$ ) of wax per gram of needle tissue and seedlings from the cross P327 $\times$ P327 had a mean of $3.7 \times 10^{-3} \mathrm{~g}\left(\mathrm{SD}=5.5 \times 10^{-2} \mathrm{~g}\right)$ of wax per gram of needle tissue. Among secondary needles, H111 had a mean of $4.7 \times 10^{-3} \mathrm{~g}\left(\mathrm{SD}=7.9 \times 10^{-4} \mathrm{~g}\right)$ of wax per gram of needle tissue and P327 had a mean of $8.7 \times 10^{-3} \mathrm{~g}$ of wax per gram $\left(2.8 \times 10^{-2} \mathrm{~g}\right)$ of needle tissue.

Stomatal wax occlusion comparisons. ESEM comparisons of the needle surfaces revealed that on the needle surface of H111 (Fig. 2A) the crystalline wax was only found around stomata and often did not occlude the stomatal openings, whereas P327 had crystalline wax that was occluding stomatal openings (Fig. 2B). Statistically significant differences $(P<0.05)$ were observed for stomatal wax occlusion between families but not primary versus secondary needles, suggesting that stomatal wax occlusion is genetically, but not developmentally, regulated in these two families (Fig. 3A). The mean percentage of stomata that were occluded on secondary needles of clone $\mathrm{P} 327$ was $98.75 \%$ (SD = 1.25\%) compared with that of susceptible clone H111 which had $48.75 \%$ $(\mathrm{SD}=6.93 \%)$ occluded. Primary needles of both selfed crossed and open-pollinated seedlings displayed similar characteristics with $97.5 \%(\mathrm{SD}=1.64 \%)$ and $42.50 \%(\mathrm{SD}=7.01 \%)$ of stomata occluded, respectively, for selfed crossed P327 and H111 seedlings and $82.5 \%(\mathrm{SD}=3.66 \%)$ and $1.00 \%(\mathrm{SD}=0.24 \%)$ of stomata occluded for open-pollinated crosses of P327 and H109, respectively.

Needles from P327 $\times$ H109 that became infected 6 weeks after inoculation had a significantly $(P \leq 0.05)$ lower percentage $(19.82 \% ; \mathrm{SD}=0.99 \%)$ of stomata that were occluded compared
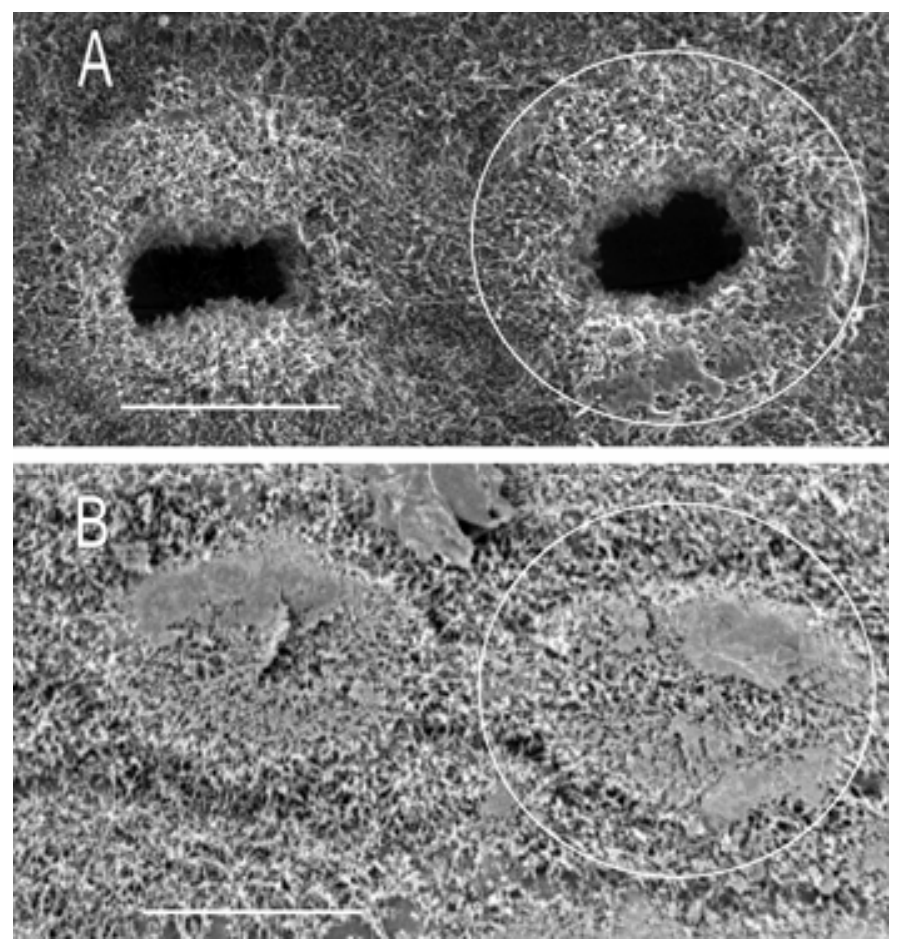

Fig. 2. Environmental scanning electron microscopy image of stomata of secondary needles of $\mathbf{A}$, susceptible clone H111 (bar $=60 \mu \mathrm{m})$ and $\mathbf{B}$, resistant clone P327 (bar $=60 \mu \mathrm{m})$. Notice the large opening to stomatal chamber and lack of wax occlusion in $\mathbf{A}$ and the complete wax occlusion of stomata in $\mathbf{B}$. White circles provide outline of a stoma. 
with needles from seedlings of the same cross that failed to develop symptoms (65.77\%; $\mathrm{SD}=1.66 \%$ ) (Fig. 3B). Infected P327 × H109 and noninfected, open-pollinated H109 needles had significantly fewer occluded stomata than other groups, but did not differ from each other. Open-pollinated P327 needles that were noninfected had the highest percentage of stomata occluded and P327 × H109 did not differ significantly from either the highest or lowest group.

Spore germination and stomatal penetration. $C$. ribicola basidiospore germination percentage differed significantly $(P \leq$ 0.05 ) on both primary and secondary needles of P327 versus susceptible H111, resistant host $P$. wallichiana (Himalayan white pine) and nonhost M. glyptostroboides (Figs. 4A, 5, and 6).

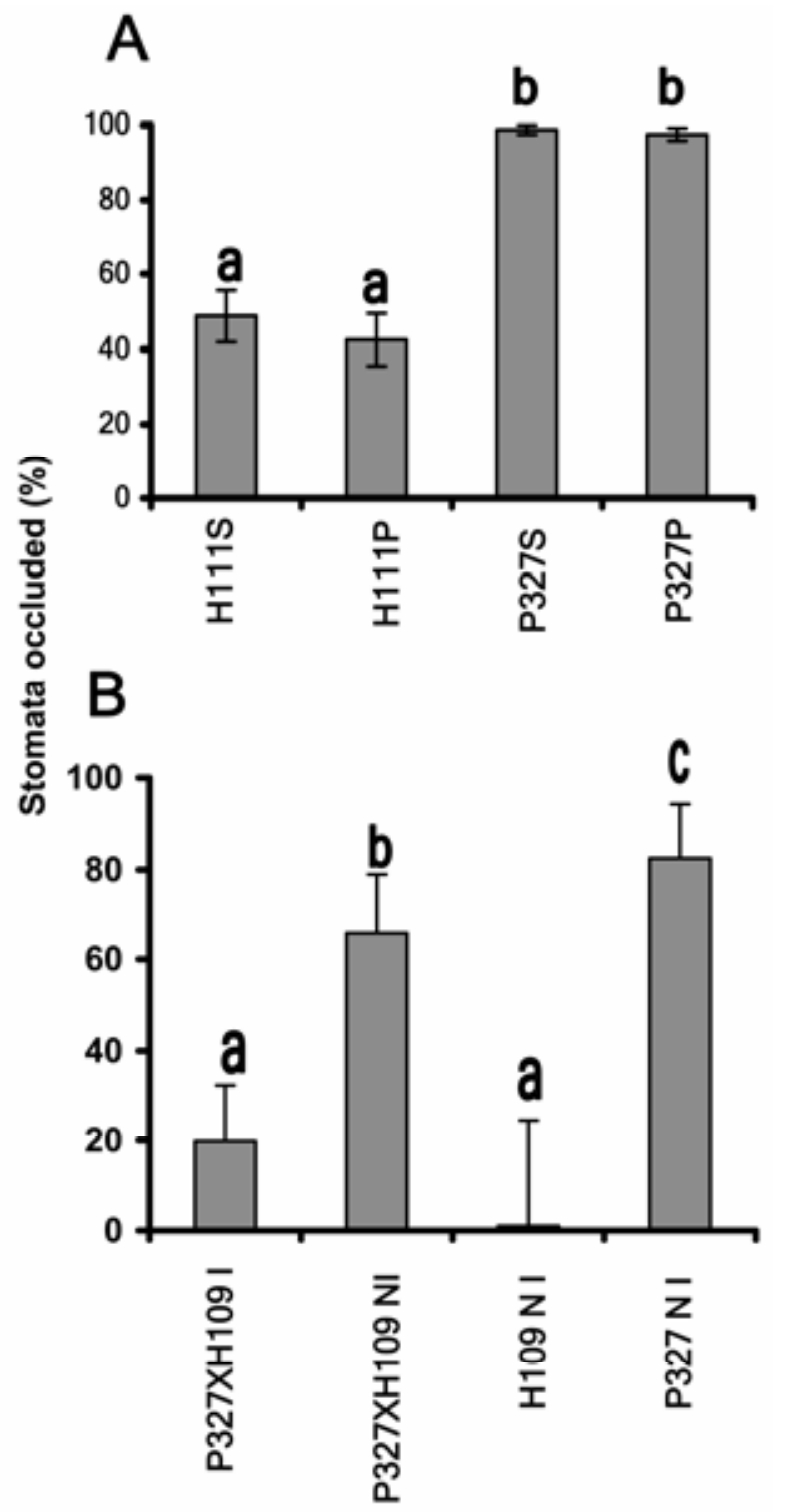

Needle type

Fig. 3. A, Average percentage of stomata occluded $(n=64)$ of primary (P) needles from seedlings of selfed crosses of H111 and P327 and secondary (S) needles from grafts of clones P327 and H111. Error bars show standard deviation and lowercase letters in bold above bars denote statistically different homogenous subsets $(\alpha=0.05$ level $)$ from Waller-Duncan test. B, Average percentage of stomata $(n=330)$ of infected (I) and noninfected (NI) primary needles from seedlings of cross P327 $\times$ H109 occluded with wax. Average wax occlusion $(n=330)$ of primary needles from seedlings of selfed crosses of H109 and P327 are also represented. Lowercase letters in bold above bars denote statistically different homogenous subsets $(\alpha=0.05$ level $)$ from Waller-Duncan test.
Significantly fewer spores germinated on P327 primary needles $(12.50 \%$ [SD $=4.91 \%])$ or secondary needles $(28.75 \%$ [SD = $6.93 \%])$ than on $\mathrm{H} 111$ primary needles $(97.50 \%$ [SD $=2.50 \%])$ or secondary needles $(91.25 \%$ [SD $=8.75 \%]), M$. glyptostroboides
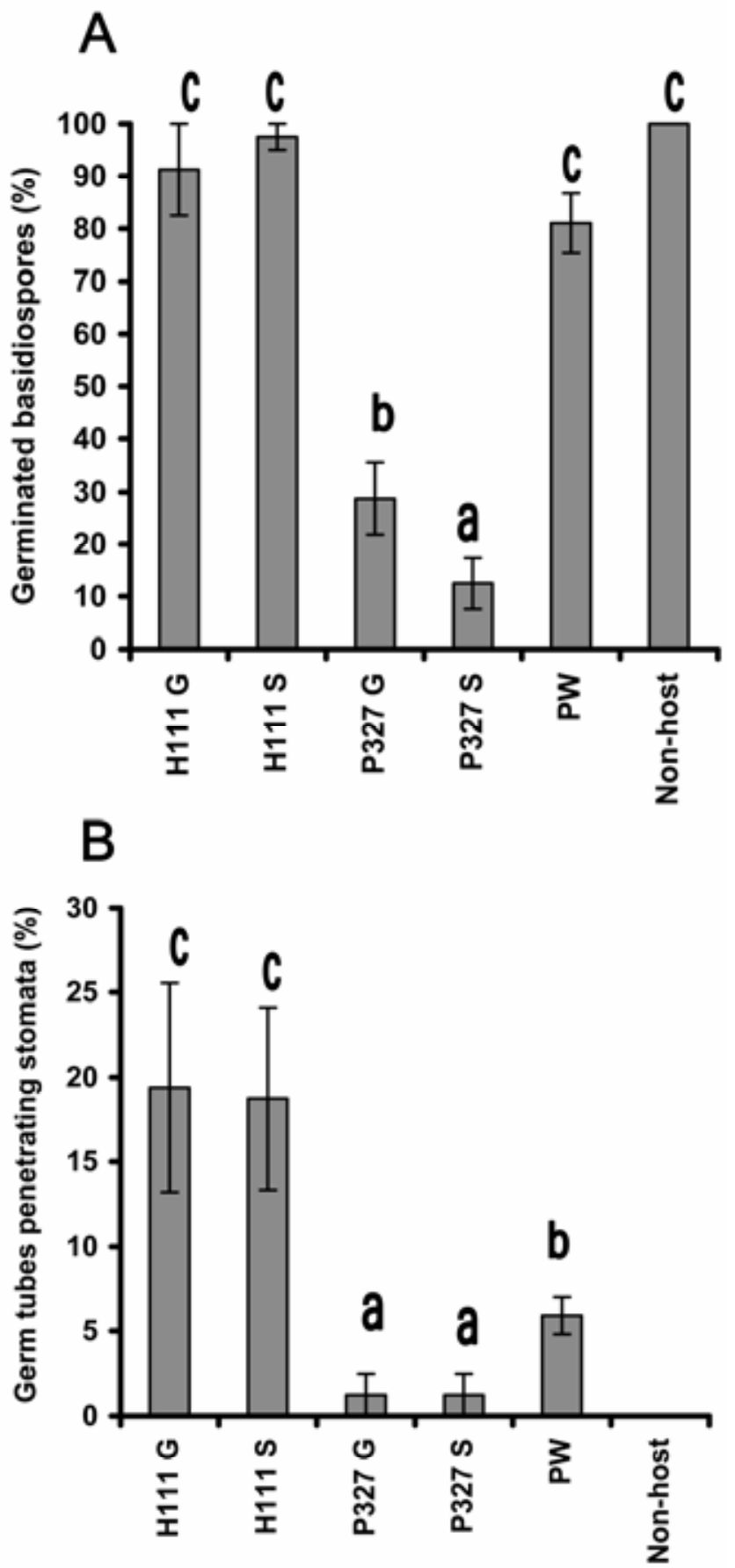

\section{Needle type}

Fig. 4. A, Average percentage of Cronartium ribicola basidiospores germinating on primary needles $(n=30)$ from seedlings of selfed crosses of H111 and P327, secondary needles $(n=30)$ from grafts of clones P327 and H111, and primary needles from resistant host Pinus wallichiana (PW) and nonhost Metasequoia glyptostroboides. Error bars show standard deviation and lowercase letters in bold above bars denote statistically different homogenous subsets $(\alpha=$ 0.05 level) from Waller-Duncan test. B, Average percentage of $C$. ribicola basidiospores with germ tubes penetrating stomata of primary needles $(n=30)$ from seedlings of selfed crosses of H111 and P327 and secondary needles $(n=$ 30) from grafts of clones P 327 and H111. Error bars show standard deviation and lowercase letters in bold above bars denote statistically different homogenous subsets $(\alpha=0.05$ level) from Waller-Duncan test. 
$(100 \%[\mathrm{SD}=0.00 \%])$ or $P$ wallichiana primary needles $(1.00 \%$ $[\mathrm{SD}=5.70 \%])$.

Significantly fewer $(P \leq 0.05)$ germ tubes penetrated the stomata of P327 primary or secondary needles (both at $1.25 \%$ [SD = $1.25 \%])$ versus $\mathrm{H} 111$ primary $(18.75 \%$ [SD $=5.40 \%])$ or secondary needles $(19.38 \%$ [SD $=6.16 \%])$, or P. wallichiana $(5.91 \%$ $[\mathrm{SD}=1.11 \%]$ ) and none had penetrated stomata on nonhost M. glyptostroboides (Figs. 4B and 7).

Wax removal inoculation. The wax removal protocol resulted in a relatively undamaged, wax-free epidermis compared with the controls (Fig. 8). Mean spot number index was significantly different only between P327 × P327 with wax present and H111 $\times$ H111 with no wax (Table 1). There was no significant difference between groups for mean spot size. P327 × P327 with wax had significantly fewer needles with infections than the other groups.

GC/MS comparison of extracted needle wax. GC/MS analyses revealed at least two peaks that were in the P327 family on both primary and secondary needles that were not present in needles from susceptible family H111 (Fig. 9). However, secondary needles of $\mathrm{H} 111$ had peaks that were not found in primary needles of H111, but were found in P327.

\section{DISCUSSION}

The results obtained from these studies provide substantial evidence for epicuticular wax playing a major role in blister-rust resistance in $P$. strobus family P327. It was observed in this study that significant differences in wax occlusions occur between a resistant and a susceptible family and that the suggestion of Patton and Spear (15) indicating that wax occlusion is likely the reason fewer infections become established on these needles is corroborated by our research. This is especially true since many spores
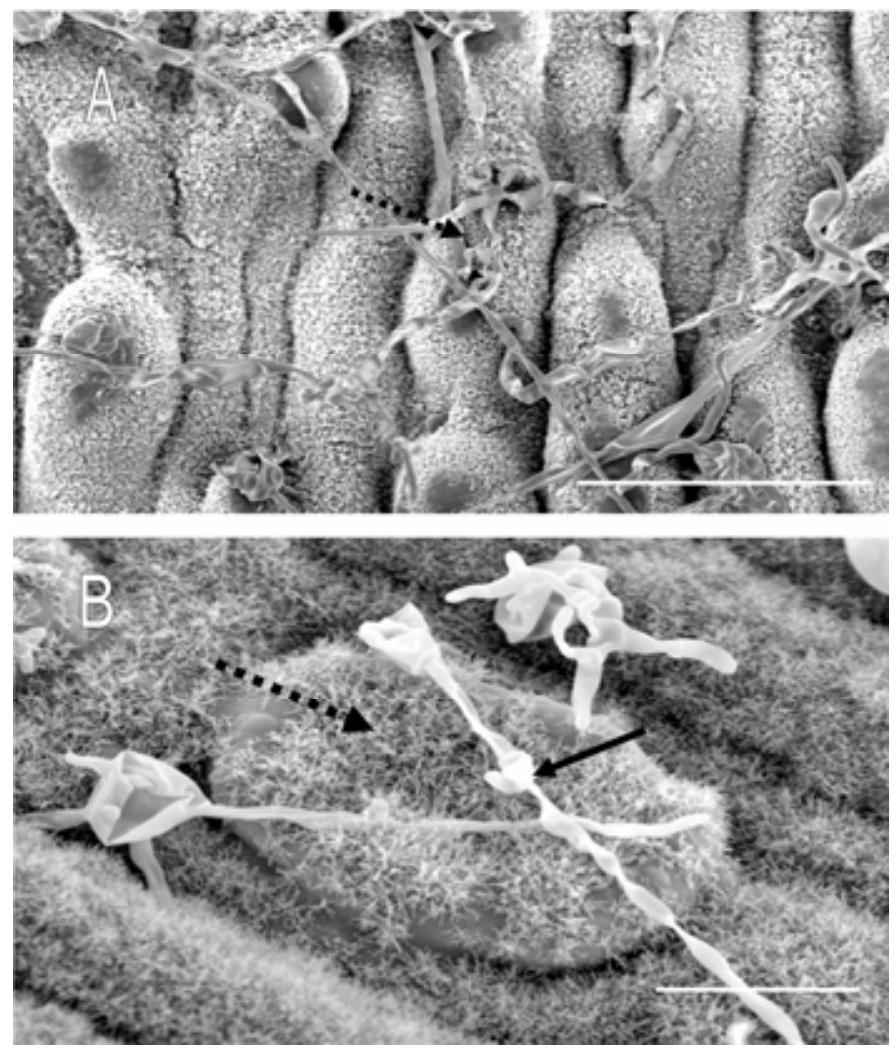

Fig. 5. Environmental scanning electron microscopy image of germinating basidiospores of Cronartium ribicola on needles of $\mathbf{A}$, nonhost Metasequoia glyptostroboides $(\mathrm{bar}=50 \mu \mathrm{m})$ and $\mathbf{B}$, resistant host Pinus wallichiana $(\mathrm{bar}=$ $20 \mu \mathrm{m}$ ). Notice the prolific germination in $\mathbf{A}$ (dashed arrow) and the hyphae crossing the stomata (dashed arrow), but not entering the stomatal chamber in B. Also note the wax-occluded stoma in $\mathbf{B}$ (solid arrow). were observed germinating on P327 with hyphae that grew across the occluded stomata, but this phenomenon was not commonly encountered for H111. Instead, there were many instances of hyphae penetrating the stomatal openings that were free of wax.

Our results also show that significant differences in spore germination and subsequent stomatal entry by $C$. ribicola occur between susceptible and resistant families and between primary and secondary needles in the resistant family P327, but these differences were not observed for susceptible family H111. Although Patton and Spear (15) found that substomatal vesicle formation was inhibited in some cases, no previous studies have analyzed wax chemistry in relation to resistance or the effect of epistomatal wax on basidiospore germination and subsequent hyphal development. Our GC/MS analyses indicate differences in wax composition exist between families, with P327 having at least two specific peaks not found in H111. A more comprehensive characterization of these differences and identification of the compounds needs to be completed in order to elucidate the role of wax chemistry and the factors that inhibit basidiospore germination.

Several studies in the past have suggested an apparent relationship between epistomatal wax and lack of penetration by developing hyphae of $C$. ribicola $(4,13,15)$. Our results showing a difference in spore germination/germ tube length as well as biochemical differences in needle wax provide further evidence for a needle surface-based resistance trait. However, chemistry alone may not confer resistance. For example, differences in wax physi-
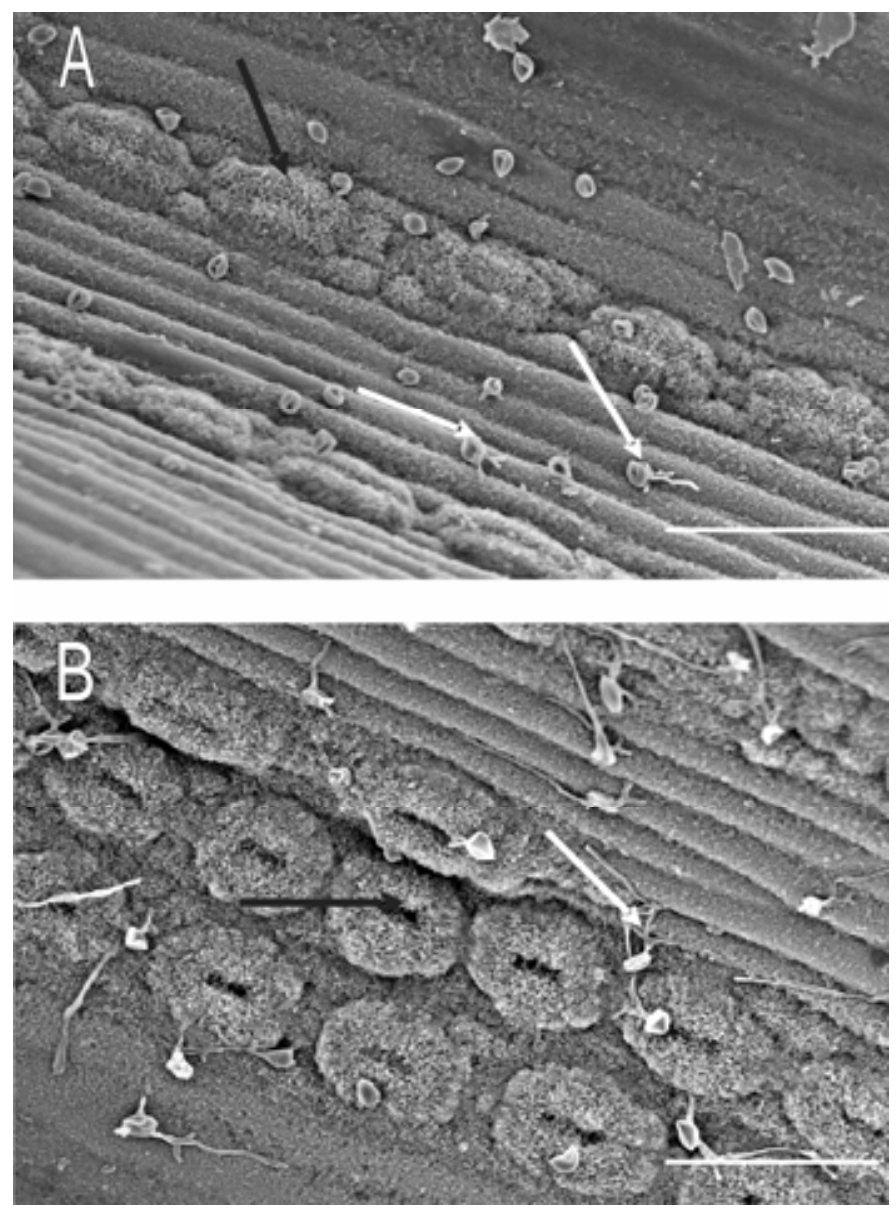

Fig. 6. Environmental scanning electron microscopy image of germinating basidiospores of Cronartium ribicola on primary needles from seedlings of $\mathbf{A}$, resistant P327 $\times$ P327 (bar $=100 \mu \mathrm{m})$ and $\mathbf{B}$, susceptible H111 $\times$ H111 (bar = $100 \mu \mathrm{m}$ ). Notice the few germinating spores with short germ tubes (white arrow) and the wax-occluded stomata (black arrow) in $\mathbf{A}$ and the numerous germinating spores with longer germ tubes (white arrow) and lack of wax occlusion of stomata (black arrow). 

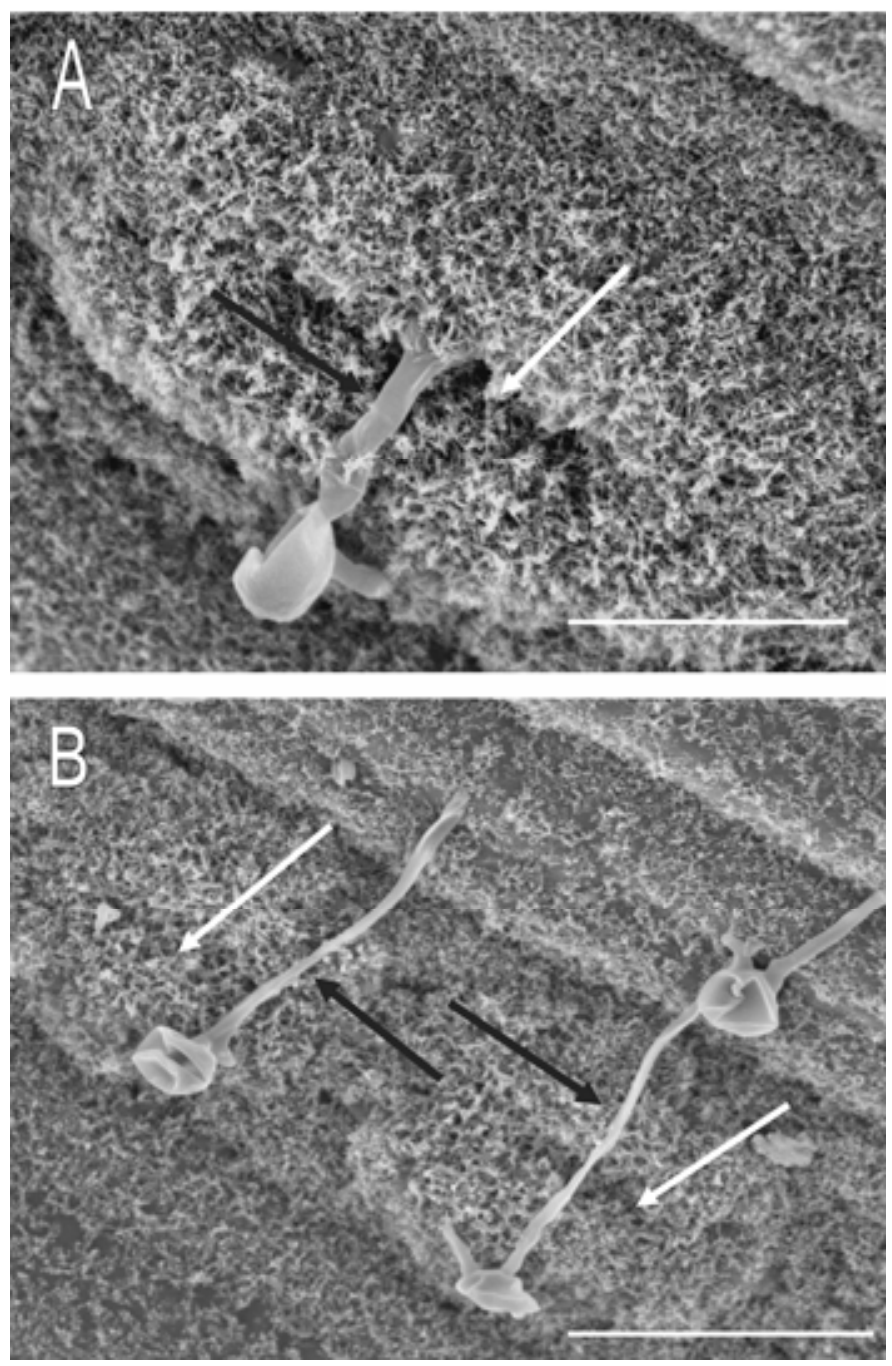

Fig. 7. Environmental scanning electron microscopy image of germinating basidiospores of Cronartium ribicola on primary needles from seedlings of A, susceptible $\mathrm{H} 111 \times \mathrm{H} 111(\mathrm{bar}=25 \mu \mathrm{m})$ and $\mathbf{B}$, resistant P327 $\times \mathrm{P} 327(\mathrm{bar}=$ $50 \mu \mathrm{m}$ ). Notice the hypha (black arrow) entering the gap in the wax (white arrow) in the stomatal chamber in $\mathbf{A}$ and the hyphae crossing the stomata (black arrows), but not entering the stomatal chambers occluded with wax (white arrows) in $\mathbf{B}$.

cal characteristics may be responsible for changes in surface water tension (free water was present on needle surfaces during inoculation), thus affecting spore germination behavior. However, further studies are needed to substantiate this. Woo et al. (17) reported that other stomatal characteristics such as stomatal size and shape may influence blister-rust resistance in P. monticola (western white pine) and possible differences in wax surface textures and/or chemistry may play a role in resistance. Their finding that surface droplet contact angles differed significantly between resistant and seed orchard bulk lots suggests a link between wax morphology and needle surface "microclimate"-one that might potentially alter basidiospore behavior.

A previous study suggested that because wax deposits occlude the stomatal antechambers, the developing hyphae are prevented from entering and subsequent development of the substomatal vesicles is aborted (15). This has not been previously substantiated through observations. The results from our study indicate that wax deposits are at least partially responsible for the "reduced needle-lesion frequency" resistance observed by Patton and Spear (15). Although SEM studies showed conclusively that hyphae can be inhibited from entering occluded stomata, it is still unclear how stomatal occlusion is responsible for resistance. For example, does stomatal occlusion alter needle surface microcli-
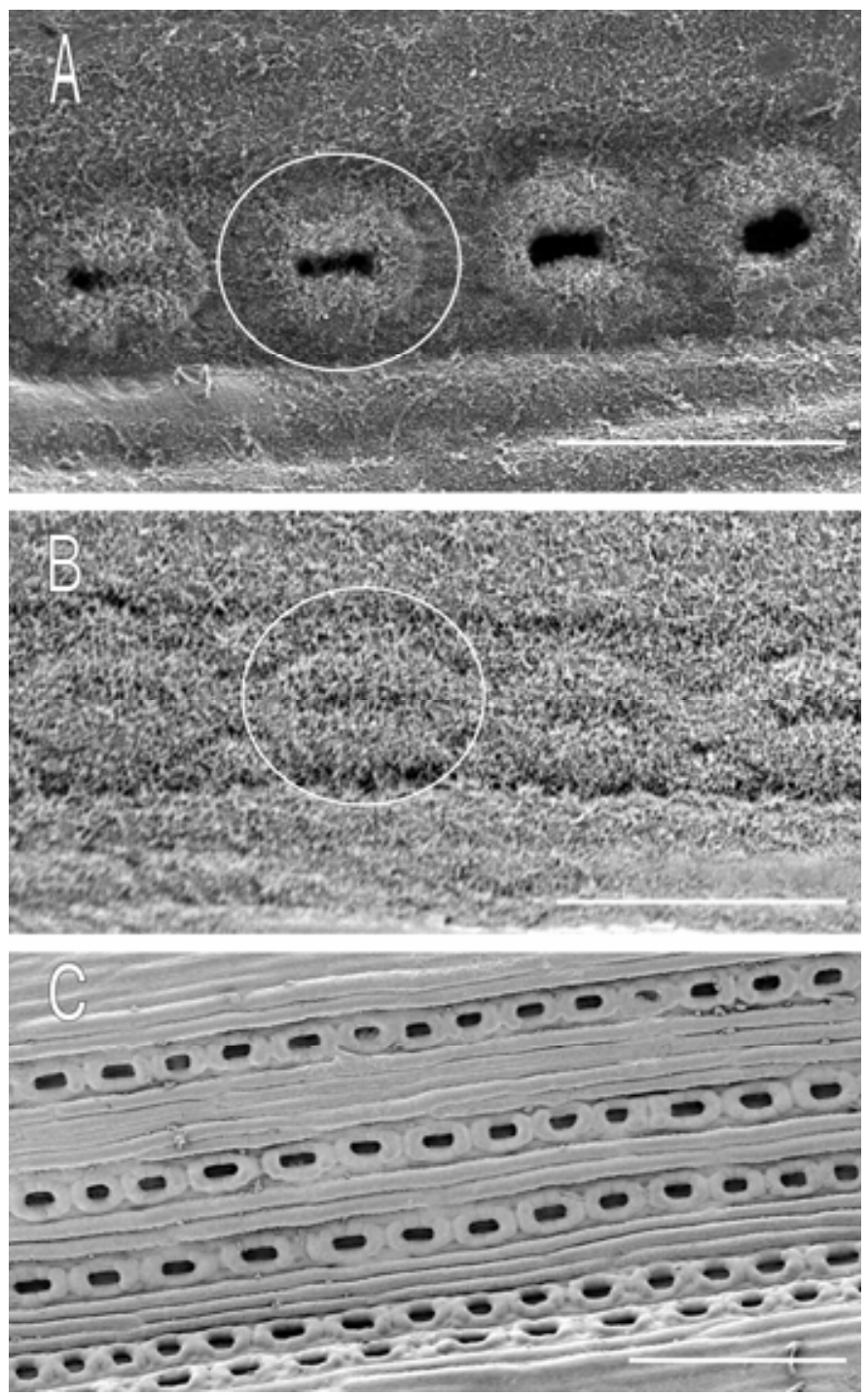

Fig. 8. Environmental scanning electron microscopy image of stomata of primary needles of $\mathbf{A}$, susceptible family $\mathrm{H} 111 \times \mathrm{H} 111($ bar $=100 \mu \mathrm{m})$, $\mathbf{B}$, resistant family P327 $\times$ P327 (bar $=100 \mu \mathrm{m}$ ), and $\mathbf{C}, \mathrm{H} 111$ after wax has been removed for inoculation $(\mathrm{bar}=20 \mu \mathrm{m})$. Notice the large opening to stomatal chamber and lack of wax occlusion in $\mathbf{A}$, the complete wax occlusion of stomata in $\mathbf{B}$, and wax-free epidermis in $\mathbf{C}$. White circles provide outline of a stoma.

TABLE 1. Average number of primary needles infected per seedling, average spot number index, and average spot size index of Pinus strobus seedlings inoculated with Cronartium ribicola ${ }^{\mathrm{z}}$

\begin{tabular}{lccc}
\hline Seedling group & $\begin{array}{c}\text { No. of needles } \\
\text { infected }\end{array}$ & Spot index & Spot size \\
\hline P327W & $7.58( \pm 1.81) \mathrm{a}$ & $2.00( \pm 0.25) \mathrm{a}$ & $2.42( \pm 1.00) \mathrm{a}$ \\
P327NW & $16.58( \pm 4.64) \mathrm{b}$ & $2.75( \pm 0.37) \mathrm{ab}$ & $2.83( \pm 0.58) \mathrm{a}$ \\
H111W & $14.08( \pm 3.88) \mathrm{b}$ & $2.75( \pm 0.37) \mathrm{ab}$ & $2.67( \pm 0.78) \mathrm{a}$ \\
H111NW & $15.83( \pm 4.07) \mathrm{b}$ & $3.00( \pm 0.25) \mathrm{b}$ & $2.75( \pm 1.00) \mathrm{a}$ \\
\hline
\end{tabular}

${ }^{\mathrm{z}} \mathrm{NW}=$ wax removed; $\mathrm{W}=$ wax not altered. Standard deviation is given in parentheses and lowercase letters within columns denote statistically different homogenous subsets ( $\alpha=0.05$ level) according to Waller-Duncan test. $n=8$.

mate and does this result in altered disease development, or is the stomatal occlusion simply a physical barrier that prevents entry by the pathogen?

One limitation to studying the relation between wax occlusion and the infection process has been the process of tissue fixation during preparation for electron microscopy. Traditional SEM 

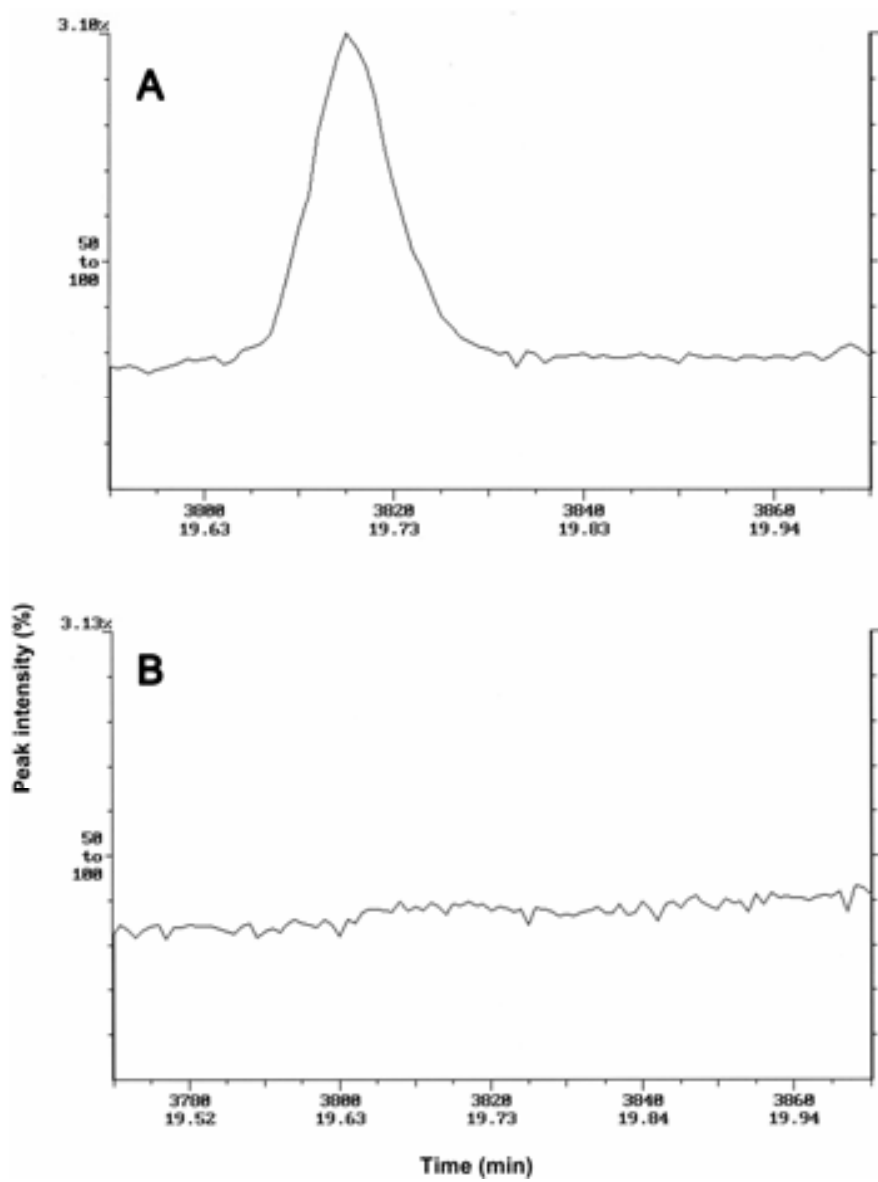

Fig. 9. Gas chromatography/mass spectrometry (GC/MS) chromatograms showing peak of a compound found in epidermal wax extracted from primary needles of $\mathbf{A}$, resistant family P327, but not found in extracted wax from primary needles from $\mathbf{B}$, susceptible family H111. GC/MS chromatograms using secondary needles provided similar results. $x$ axis $=$ time (minutes) and $y$ axis $=$ peak intensity.

protocols use solvents that destroy needle wax (15). Thus, it has not been possible to observe germinating spores and the needle surface as it appears in vivo. Only recently, with the advent of ESEM, has this become possible.

It is important to note that although genetics play a clear role in differences in wax development among $P$. strobus genotypes, other factors such as growing environment (nutrient availability, atmospheric conditions such as pollution, etc.) may also influence wax development and characteristics in conifers. For example, Chiu et al. (2) showed that fertilization did not change quantity of needle wax in Pseudotsuga menziesii (Douglas fir), but did change glaucousness caused by ornate tubular epicuticular wax in nonstomatal regions. Thus, there are likely interactions of the environment and host genetics that either favor or discourage disease development.

These studies provide important new information on early infection processes of $P$. strobus families by $C$. ribicola. Results indicate that resistant family P327 has multiple traits that confer resistance - i.e., ability to inhibit spore germination (possibly due to wax chemistry) and capacity to inhibit entry of stomata by C. ribicola hyphae due to waxes that occlude the stomata. Identification of traits (such as those reported here) that confer resis- tance to $C$. ribicola in $P$. strobus provides valuable criteria for selecting for resistance to WPBR, potentially significantly circumventing years of breeding, inoculations, and screening individuals.

\section{ACKNOWLEDGMENTS}

We thank the Cloquet Forestry Center, University of Minnesota and the Oconto River Seed Orchard, U.S. Forest Service for their help in acquiring plant materials used in this study. We also thank G. Ahlstrand at the Imaging Center, College of Biological Sciences, University of Minnesota for his aid with environmental scanning electron microscopy and T. Krick at the Mass Spectrometry Consortium for the Life Sciences, College of Biological Sciences, University of Minnesota for helping with GC/MS analyses. Funds for some aspects of this research were provided by the Minnesota Tree Improvement Cooperative, University of Minnesota.

\section{LITERATURE CITED}

1. Boyer, M. G., and Isaac, P. K. 1964. Some observations on white pine blister rust as compared by light and electron microscopy. Can. J. Bot. 42:1305-1309.

2. Chiu, S.-T., Anton, L. H., and Ewers, F. W., Hammerschmidt, R., and Pregitzer, K. S. 1992. Effects of fertilization on epicuticular wax morphology of needle leaves of Douglas fir, Pseudotsuga menziesii (Pinaceae). Am. J. Bot. 79:149-154

3. Hansen, E. M., and Patton, R. F. 1975. Types of germination and differentiation of vesicles by basidiospores of Cronartium ribicola. Phytopathology 65:1061-1071

4. Hansen, E. M., and Patton, R. F. 1977. Factors important in artificial inoculation of Pinus strobus with Cronartium ribicola. Phytopathology 67:1108-1112.

5. Heimburger, C. 1962. Breeding for disease resistance in forest trees. Forest. Chron. 38:356-362.

6. Hirt, R. B. 1938. Relation of stomata to infection of Pinus strobus by Cronartium ribicola. Phytopathology 28:180-190.

7. Jurgens, J. A., Blanchette, R. A., Zambino, P. J., and David, A. 2003. Histology of white pine blister rust in needles of resistant and susceptible eastern white pine. Plant Dis. 87:1026-1030.

8. Kinloch, B. B., Jr., Sniezko, R. A., Barnes, G. D., and Greathouse, T. E. 1999. A major gene for resistance to white pine blister rust in western white pine from the Western Cascade range. Phytopathology 89:861-867.

9. Maloy, O. C. 2001. White pine blister rust. Online. Plant Health Progress doi: 10.1094/PHP-2001-0924-01-HM

10. Patton, R. F. 1961. The effect of age upon susceptibility of eastern white pine to infection by Cronartium ribicola. Phytopathology 51:429-434.

11. Patton, R. F. 1967. Factors in white pine blister rust resistance. Pages 876890 in: Proc. XIVth IUFRO Congress. Part III, Section 22 and Intersectional Working Group 22/24.

12. Patton, R. F. 1972. Inoculation methods and problems in testing eastern white pine for resistance to Cronartium ribicola. Pages 373-385 in: Biology of Rust Resistance in Forest Trees. U.S. Dep. Agric. For. Ser. Misc. Publ. 1221.

13. Patton, R. F., and Johnson, D. W. 1970. Mode of penetration of needles of eastern white pine by Cronartium ribicola. Phytopathology 60:977-982.

14. Patton, R. F., and Riker, A. J. 1966. Lessons from nursery and field testing of eastern white pine selections and progenies for resistance to blister rust. Pages 403-420 in: Breeding Pest-Resistant Trees. Proc. NATO and NSF Symposium. H. D. Gerhold, E. J. Schreiner, R. E. McDermott, and J. A. Winieski, eds. Pergamon Press, New York.

15. Patton, R. F., and Spear, R. N. 1980. Stomatal influences on white pine blister rust infection. Phytopathol. Mediterr. 19:1-7.

16. Riker, A. J., Kouba, T. F., Brener, W. H., and Patton, R. F. 1953. White pine trees selected for resistance to white pine blister rust. Proc. 7th Int. Botanical Congress 14:48.

17. Woo, K.-S., Fins, L., McDonald, G. I., and Wiese, M. V. 2001. Differences in needle morphology between blister rust resistant and susceptible western white pine stocks. Can. J. For. Res. 31:1880-1886.

18. Zambino, P. J., and Michler, C. H. 1999. Accelerated identification of eastern white pine families resistant to white pine blister rust. (Abstr.) Phytopathology 89(suppl.):S89. 